Scientific Paper

\title{
Determining optimum wavelength of ultraviolet rays to pre-exposure of non-uniformity error correction in Gafchromic EBT2 films
}

\author{
Toshizo KATSUDA ${ }^{1, a}$, Rumi GotANDA ${ }^{2}$, Tatsuhiro GotANDA ${ }^{2}$, Takuya AKAGAWA ${ }^{3}$, Nobuyoshi TANKI ${ }^{1}$, Tadao \\ $\mathrm{KUWANO}^{4}$, Atsushi NOGUCHI ${ }^{1}$, Kouichi YABUNAKA ${ }^{5}$ \\ ${ }^{I}$ Faculty of Health Sciences, Butsuryo College of Osaka, 3-33, Otorikita-machi Nishi-ku, Sakai-city, Osaka, 593-8328, Japan \\ ${ }^{2}$ Faculty of Health Science and Technology, Kawasaki University of Medical Welfare, Okayama, Japan \\ ${ }^{3}$ Department of Radiological Technology, Tokushima Red Cross Hospital, 103, Irinokuchi, Komatsujshima, Tokushima, 773 - \\ 8502, Japan \\ ${ }^{4}$ Graduate School of Health Sciences, Okayama University, 2-5-1, Shikata-cyo, Kita-ku, Okayama-city, Okayama, 700-8558, \\ Japan \\ ${ }^{5}$ Graduate School of Medicine, the University of Tokyo, 7-3-1 Hongo, Bunkyou-Ku, Tokyo, 113-0033, Japan \\ ${ }^{a}$ E-mail address: tkatsudajp@yahoo.co.jp
}

(received 28 September 2017; revised 20 December 2017; accepted 6 February 2018)

\begin{abstract}
Gafchromic films have been used to measure X-ray doses in diagnostic radiology such as computed tomography. The double-exposure technique is used to correct non-uniformity error of Gafchromic EBT2 films. Because of the heel effect of diagnostic x-rays, ultraviolet A (UV-A) is intended to be used as a substitute for x-rays. When using a UV-A light-emitting diode (LED), it is necessary to determine the effective optimal UV wavelength for the active layer of Gafchromic EBT2 films. This study evaluated the relation between the increase in color density of Gafchromic EBT2 films and the UV wavelengths. First, to correct non-uniformity, a Gafchromic EBT2 film was pre-irradiated using uniform UV-A radiation for $60 \mathrm{~min}$ from a $72-\mathrm{cm}$ distance. Second, the film was irradiated using a UV-LED with a wavelength of 353-410 $\mathrm{nm}$ for $60 \mathrm{~min}$ from a $5.3-\mathrm{cm}$ distance. The maximum, minimum, and mean \pm standard deviation (SD) of pixel values of the subtraction images were evaluated using 0.5 inches of a circular region of interest (ROI). The highest mean \pm SD $(8915.25 \pm 608.86)$ of the pixel value was obtained at a wavelength of $375 \mathrm{~nm}$. The results indicated that $375 \mathrm{~nm}$ is the most effective and sensitive wavelength of UV-A for Gafchromic EBT2 films and that UV-A can be used as a substitute for x-rays in the double-exposure technique.
\end{abstract}

Key words: ultraviolet ray wavelength; gafchromic films; computed tomography; diagnostic radiology; doubleexposure technique.

\section{Introduction}

Gafchromic films have been used for quality assurance, quality control, and radiation dose measurement in diagnostic examinations such as computed tomography [1-4]. Gafchromic EBT films are designed to measure absorbed doses of $\mathrm{x}$-rays with minimal energy dependence [5]. The double-exposure technique is used in therapeutic radiology to correct the nonuniformity of the active layer of Gafchromic EBT films [6]. However, this technique uses UV-A rather than X-rays because uniform irradiation in diagnostic examination is difficult due to the heel effect of $\mathrm{x}$-rays.

A previous study showed that a UV-A peak wavelength of $365 \mathrm{~nm}$ was most effective for Gafchromic EBT2 films, rather than UV-B or UV-C [7]. However, the specific wavelength (narrow bandwidth) of UV-A is still unclear. The UV-A bandwidth ranged from 315 to $400 \mathrm{~nm}$ [8] and a 10-W fluorescent UV lamp was used as a UV-A source. In addition,
UV-A pre-irradiation corrected the non-uniformity of Gafchromic EBT2 films [9].

The EBT2 film works on the basis of solid-state topochemical polymerization reaction initiated by irradiation (UV-A). The color of the film changes from light yellow to dark blue [5]. However, only the front side of the film is protected from UV irradiation [7]; the reaction of the active layer is observed when the back side of the film is irradiated with UV-A.

In this study, a UV-A light-emitting diode (LED: Nitride Semiconductors Co., Ltd., Tokushima, Japan) with a wavelength of 353-400 nm was used, which has a narrow bandwidth compared with fluorescent UV lamps, emitting photons in range of approximately $310 \mathrm{~nm}$ to $410 \mathrm{~nm}$ [10]. The most effective UV-A wavelength was determined during our investigation. This study aimed to determine the optimal UV-A wavelength using a UV-LED as a substitution for $\mathrm{x}$-rays in the double-exposure technique for Gafchromic EBT2. 


\section{Materials and Methods}

\section{UV-LED}

In this study, a UV-LED that emitted UV-A rays at a wavelength of $353 \mathrm{~nm}$, with $5 \mathrm{~nm}$ increments from 360 to $400 \mathrm{~nm}$, was used. Table 1 shows the representative characteristics of UV-LED, and Figure 1 shows UV-LED that was used in this study.

\section{Gafchromic film}

We used a $10.2 \mathrm{~cm} \times 25.4 \mathrm{~cm}$ Gafchromic EBT2 film (lot \#02171403; Ashland, Inc., Covington, KY, USA).

\section{UV-A Irradiation device}

A UV exposure acrylic plate (Comogras CG UV40 P, 3-mm thick, lot \#140406C B; Kuraray Co., Ltd., Tokyo, Japan) containing 20 holes with a diameter of $30 \mathrm{~mm}$ each (Figure 2) was used for fixing the irradiation position of the Gafchromic EBT2 film. Because UV rays are known to cause cellular damage [10], a UV irradiation box was constructed using UVcut acrylic board to prevent any radiation leakage from the box. UV-LED was attached to this acrylic box (Figure 3). A schematic of the setup is shown in Figure 4.

\section{Irradiation method}

The Gafchromic EBT2 film was fixed onto the 3-mm-thick acrylic plate to improve the reproducibility of scanned images. First, the entire film was uniformly irradiated with UV-A for 60 min using a 10-W UV fluorescent lamp at a distance of $72 \mathrm{~cm}\left(69.4 \mu \mathrm{W} / \mathrm{cm}^{2} \times 300 \mathrm{~s}=20.82 \mathrm{~mJ} / \mathrm{cm}^{2}\right)$ [7]. Next, the film was irradiated through an irradiation hole using UV-LED at different wavelengths for 60 min each from a distance of 5.3 $\mathrm{cm}$.

Table 1. Characteristics of UV-LED $\left(I_{f}\right.$ - direct current (DC), $\lambda_{p}$ peak wavelength, Int - interpolated values, $V_{f}-$ voltage, PO photo output.)

\begin{tabular}{ccccc}
\hline \hline LED Name & $\boldsymbol{I}_{\mathbf{F}}(\mathbf{m A})$ & $\boldsymbol{\lambda}_{\mathbf{p}}(\mathbf{n m})$ & $\boldsymbol{V}_{\mathbf{F}}(\mathbf{V})$ & $\mathbf{P O}(\mathbf{m W})$ \\
\hline NS355L-5RLO & 20 & 353 & 3.2 & 1.2 \\
NS360L-5RLO & 20 & 360 & 3.2 & 1.8 \\
NS365L-5RLO & 20 & 365 & 3.2 & 2.4 \\
NS370L-5RLO & 20 & 370 & 3.2 & 4.0 \\
NS375L-5RLO & 20 & 375 & 3.2 & 11.0 \\
NS385L-ERLO & 20 & 380 & 3.2 & 17.4 \\
& 20 & 385 (Int.) & 3.6 & 19.2 \\
NS395L-ERLO & 20 & 390 & 3.0 & 21.0 \\
& 20 & 395 (Int.) & 3.2 & 25.2 \\
NS400L-ERLO & 20 & 400 & 3.0 & 21.0 \\
\hline \hline
\end{tabular}

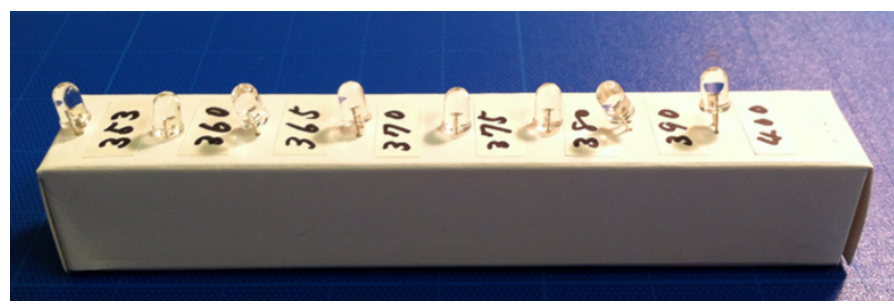

Figure 1. Output wavelengths of UV-LED ranged from 353 to 400 nm.

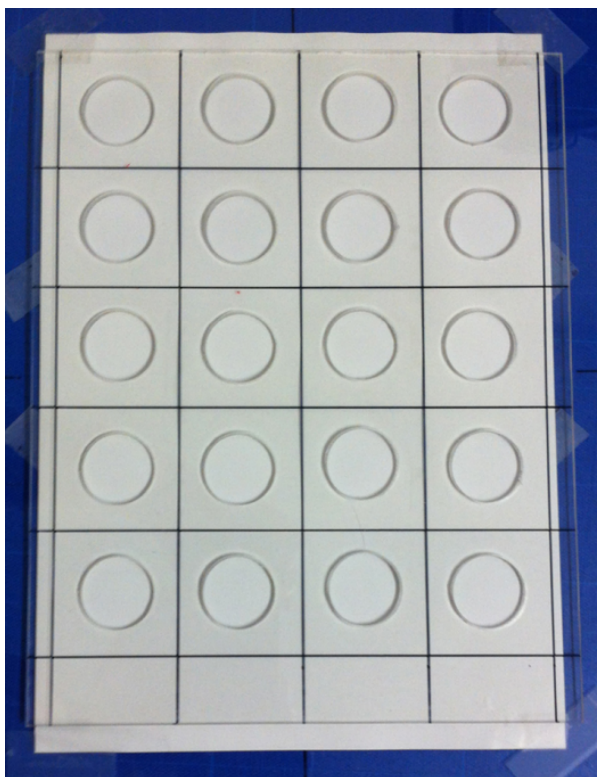

Figure 2. UV exposure plate

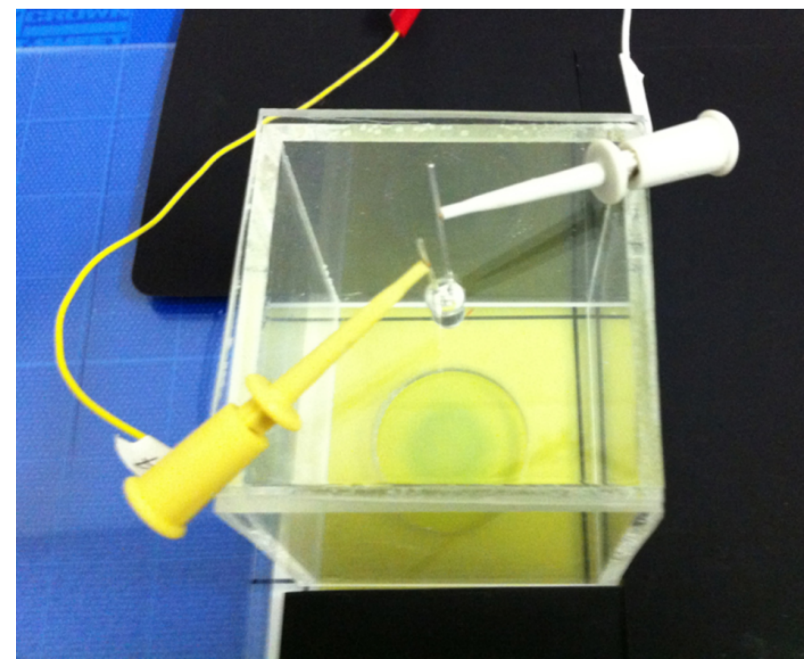

Figure 3. UV exposure device for a Gafchromic EBT2 film

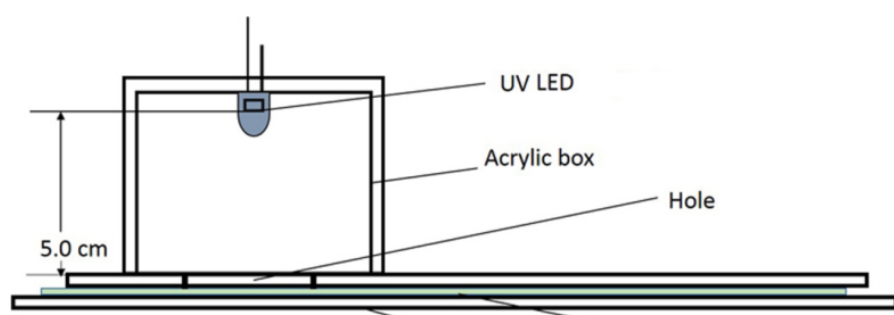

Acrylic Plate GAF

Figure 4. Schematic of the exposure setup showing UV-LED and the Gafchromic EBT2 film 


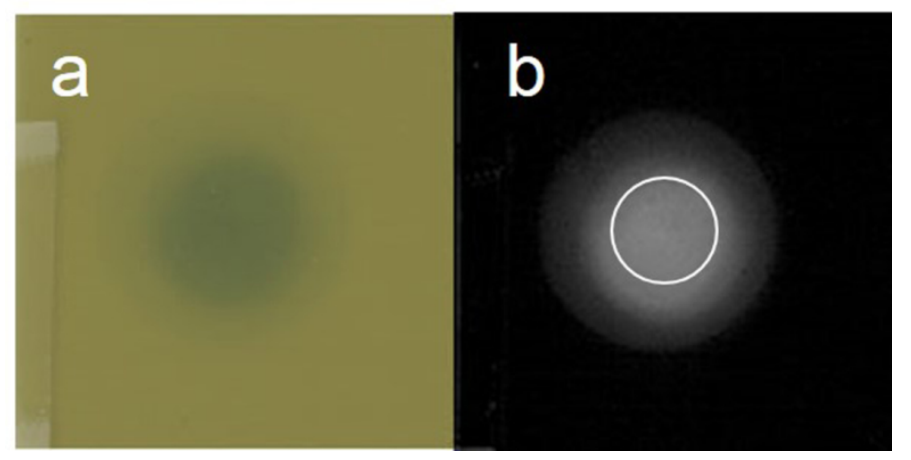

Figure 5. Colored Gafchromic EBT2 film. (a) Scanned UV exposure image of the Gafchromic EBT2 film. (b) Subtracted image with ROI

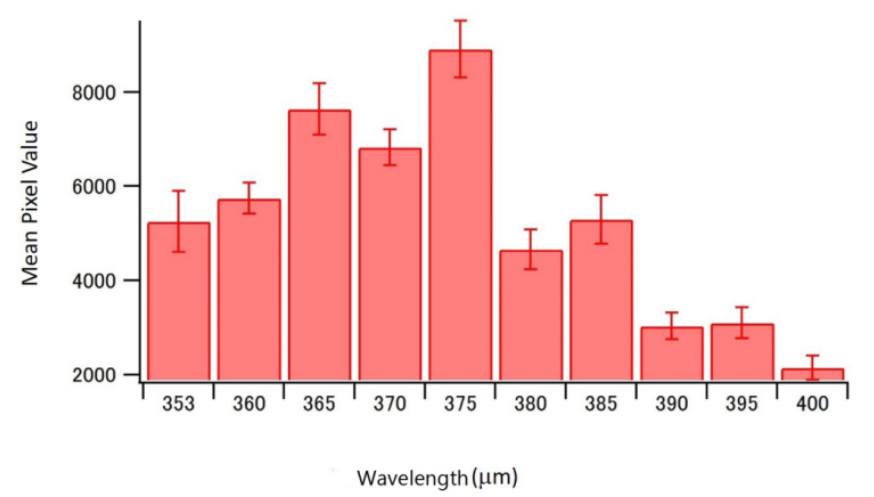

Figure 6. Graph of UV-LED measurement data for the Gafchromic EBT2 film

Table 2. Pixel values of subtracted Gafchromic EBT2 images at each $U V$ wavelength

\begin{tabular}{cccc}
\hline \hline \multirow{2}{*}{$\lambda_{\mathbf{p}}(\mathbf{n m})$} & \multicolumn{3}{c}{ Pixel values } \\
\cline { 2 - 4 } & Max & Min & Mean \pm SD \\
\hline 353 & 9035 & 0 & $5255.18 \pm 655.41$ \\
360 & 6889 & 4777 & $5746.91 \pm 326.72$ \\
365 & 9131 & 5853 & $7642.90 \pm 546.88$ \\
370 & 8501 & 5069 & $6824.28 \pm 378.18$ \\
375 & 12005 & 6972 & $8915.25 \pm 608.86$ \\
380 & 5925 & 2933 & $4653.39 \pm 430.81$ \\
385 & 7001 & 3393 & $5300.75 \pm 511.43$ \\
390 & 4053 & 1877 & $3035.42 \pm 279.83$ \\
395 & 4937 & 539 & $3100.12 \pm 319.62$ \\
400 & 3697 & 952 & $2149.11 \pm 261.96$ \\
\hline \hline
\end{tabular}

Table 3. Strength of each UV ray

\begin{tabular}{cc}
\hline \hline$\lambda_{\mathbf{p}}(\mathbf{n m})$ & UV power density $\left(\boldsymbol{\mu W} / \mathbf{c m}^{\mathbf{2}}\right)$ \\
\hline 353 & 187.07 \\
360 & 220.36 \\
365 & 507.10 \\
370 & 653.70 \\
375 & 1423.90 \\
380 & 536.20 \\
385 & 656.90 \\
390 & 85.29 \\
395 & 91.85 \\
400 & 13.90 \\
\hline \hline
\end{tabular}

\section{Scanning method}

Scanning was performed after every UV-A irradiation using a flatbed scanner (EPSON ES-10000G; Seiko Epson Corporation, Nagano, Japan). Images were acquired using Adobe Photoshop CS2 (Adobe Systems Inc., San Jose, CA, USA) at 48 bits, 100 dpi resolution, and in red, green, blue mode with a plain paper copier film (CR-PP686; 3M Company, St. Paul, MN, USA) with a protective film of liquid crystal (LCD-230W; Sanwa Supply Inc., Okayama, Japan) for the removal of Moiré artifacts (Newton rings) [2]. The temperature was maintained at $21^{\circ} \mathrm{C}-25^{\circ} \mathrm{C}$ during irradiation and image acquisition.

\section{Data analysis}

The red channel image was used for analysis. A circular region of interest (ROI) of $1.27 \mathrm{~cm}$ diameter for measuring pixel values was set in the center of the UV-A irradiation field (Figure 5a, and b). The pixel value of each UV wavelength was measured using Image $J$ version 1.440 image analysis software for Macintosh (National Institutes of Health, Bethesda, MD, USA). The maximum, minimum, and mean \pm standard deviation (SD) of the pixel values were evaluated.

\section{Results}

Wavelength versus pixel value were presented on Figure 6. For $375 \mathrm{~nm}$ UV LED, the maximum, minimum, and mean \pm SD of pixel values were 12005, 6972, and 8915.25 \pm 608.86 , respectively (Table 2). The UV-A power density was 1423,9 $\mu \mathrm{W} / \mathrm{cm}^{2}$ (Table 3).

\section{Discussion}

\section{Wavelength of the UV-LED}

As mentioned before, a previous study using a UV fluorescent lamp reported that a peak wavelength of $365 \mathrm{~nm}$ was most effective for Gafchromic EBT2 films [7]. However, this study showed that the maximum pixel values were obtained at a UVLED wavelength of $375 \mathrm{~nm}$. The cannonball-shaped UV-LED with a wavelength of $353 \mathrm{~nm}$ and a 5-nm interval from 360 to $400 \mathrm{~nm}$ was most suitable for this study. The bandwidth was narrower than that of the UV fluorescent lamp. Therefore, the results showed that a UV-LED is superior to a UV fluorescent lamp for irradiation of Gafchromic EBT2s with UV-A rays.

\section{Future studies}

Although the most suitable irradiation wavelength is determined to be $375 \mathrm{~nm}$, the optimal strength (irradiation duration) of UV-A is still unclear, for which further studies are necessary. 


\section{Conclusion}

The Gafchromic EBT2 film reacted most effectively to UV-A from a UV-LED at a wavelength of $375 \mathrm{~nm}$. Therefore, to correct the non-uniformity error of Gafchromic EBT2 films should be used $375 \mathrm{~nm}$ from a UV-LED substitute for x-rays in the double-exposure technique.

\section{Acknowledgements}

This study was supported by a grant from JSPS KAKENHI (Grant no.: 17K10380).

\section{References}

[1] Gotanda T, Katsuda T, Gotanda R, et al: Half-Value Layer Measurement: Simple Process Method Using Radiochromic Film. Australas Phys Eng Sci Med. 2009;32(3):150-158.

[2] Gotanda T, Katsuda T, Gotanda R, et al: Evaluation of effective energy using radiochromic film and a step-shaped aluminum filter. Australas Phys Eng Sci Med. 2011;34(2):213-222.

[3] Gotanda R, Katsuda T, Gotanda T, et al: Computed tomography phantom for radiochromic film dosimetry. Australas Phys Eng Sci Med. 2007;30(3):194-199.

[4] Katsuda T, Gotanda R. DOSE MEASURING METHOD AND PHANTOM, AND X-RAY IMAGE PICKING-UP DEVICE USED FOR THE DOSE MEASURING METHOD, WIPO Patent Application WO/2008; 087952.

[5] Lewis F. David: Radiochromic Film. Advanced Materials Group Ashland Specialty Ingredients 2014; Spain: April 2014.

[6] Zhu Y, Kirov AS, Mishra V, et al. Quantitative evaluation of radiochromic film response for two-dimensional dosimetry. Med Phys. 1997;24(2):223-231.

[7] Katsuda T, Gotanda R, Gotanda T, et al. Comparing three UV wavelengths for pre-exposing Gafchromic EBT-2 and EBT-3 films. J Appl Clin Med Phys. 2015;16(5):449-457.

[8] ISO 2007: ISO 2007 Space environment (natural and artificial)-process for determining solar irradiance. ISO. 2007; 21348 (Geneva: International Organization for Standardization).

[9] Katsuda T, Gotanda R, Gotanda T, et al. Correction of nonuniformity error of Gafchromic EBT2 and EBT3. J Appl Clin Med Phys. 2016;17(3):41-51.

[10] NEC, Data of FL10BL-B: NEC Lighting, Ltd. (in Japanese). http://www.nelt.co.jp/products/_1833.html?p_encd=06111495. Accessed December, 19, 2017.

[11] de Gruijl FR. Skin cancer and solar UV radiation Eur J Cancer. 1999; 35(14): 2003-2009. 